\title{
Medición y representación gráfica de las distancias culturales entre países latinoamericanos
}

\author{
Measurement and graphic representation of cultural distances between \\ Latin American countries
}

\author{
Pablo Farías / pfarias@fen.uchile.cl \\ Universidad de Chile, Chile
}

\begin{abstract}
Despite the central role of cultural distances in social sciences, literature is surprisingly scarce in quantitatively measuring and graphically representing this concept in Latin America. This study addresses this issue calculating a cultural distance index using the nine cultural dimensions measured by House et al. (2004) for ten Latin American countries: Argentina, Bolivia, Brazil, Colombia, Costa Rica, Ecuador, El Salvador, Guatemala, Mexico and Venezuela. Also, this study graphically presents the cultural distances calculated. The relevance of this study is the ability to incorporate cultural indicators that allow comparing the current situation and prospects in Latin America. In order to illustrate the implications of this study, the case of cultural distances of Mexico with other nine Latin American countries is analyzed.
\end{abstract}

Key words: national culture, cultural distances, cultural dimensions, Latin America.

Resumen: Pese al rol central que tienen las distancias culturales en las ciencias sociales, la literatura es sorprendentemente escasa en la medición cuantitativa y representación gráfica de este concepto en América Latina. El presente estudio aborda esta cuestión al mostrar un índice de distancia cultural usando las nueve dimensiones culturales medidas por House et al. (2004) para diez países latinoamericanos: Argentina, Bolivia, Brasil, Colombia, Costa Rica, Ecuador, El Salvador, Guatemala, México y Venezuela. También se representa gráficamente las distancias culturales calculadas. La relevancia de esta investigación radica en la posibilidad de incorporar indicadores culturales que permiten la comparación de la situación actual y perspectivas en América Latina. Con el propósito de ilustrar las implicancias de este estudio, se analiza el caso de las distancias culturales de México con los otros nueve países latinoamericanos.

Palabras clave: cultura nacional, distancias culturales, dimensiones culturales, América Latina. 


\section{Introducción}

Las diferencias entre países que se observan en la forma de pensar, actuar y reaccionar de las personas tiene un gran impacto en las distintas áreas de las ciencias sociales; debido a esto adquiere gran importancia el hecho de poder reconocer, medir cuantitativamente e interpretar las distancias culturales entre países. El concepto de distancia cultural entre éstos ha sido aplicado a una gran variedad de investigaciones, incluyendo el análisis del efecto de la distancia cultural entre países sobre el comportamiento de los inmigrantes y los expatriados, acuerdos internacionales entre naciones, cambios sociales, inversión extranjera, expansión internacional, funcionamiento de filiales extranjeras, transformación organizacional, preferencias del consumidor, efectividad de los formatos de publicidad, uso de medios y canales de distribución, aprendizaje organizacional, transferencias de tecnologías, etcétera (Shenkar, 2001; Manzur et al., 2012; Meunier y Medeiros, 2013; Olavarrieta et al., 2013; Farías, 2015).

Pese al rol central que tienen las distancias culturales entre países en las ciencias sociales, la literatura es sorprendentemente escasa en la medición cuantitativa y representación gráfica de este concepto en América Latina. El presente estudio aborda esta cuestión utilizando las nueve dimensiones culturales medidas por House et al. (2004) para diez países latinoamericanos (Argentina, Bolivia, Brasil, Colombia, Costa Rica, Ecuador, El Salvador, Guatemala, México y Venezuela) y empleando las metodologías sugeridas por Kogut y Singh (1988) y Fernández et al. (2003) para medir cuantitativamente y representar gráficamente las distancias culturales entre países en América Latina.

En este estudio se presenta una metodología para medir cuantitativamente y representar gráficamente las distancias culturales y proyectar posibles cambios en las distancias culturales entre los diez países latinoamericanos incluidos en el estudio de House et al. (2004). La relevancia de este trabajo es la posibilidad de incorporar indicadores culturales que permiten la comparación de la situación actual y perspectivas en América Latina. El resto del artículo se compone de la siguiente forma: la segunda sección expone el marco conceptual. La sección tres presenta formalmente la metodología y los resultados de la investigación. En la cuarta sección, con el propósito de ilustrar las implicancias de este estudio, se analiza el caso de las distancias culturales de México con los otros nueve países latinoamericanos. Finalmente, la quinta sección entrega las conclusiones y las implicancias de este trabajo para los investigadores y los administradores. 


\section{Marco conceptual}

\section{Cultura nacional}

Hofstede (1980) condujo en la década de 1970 uno de los estudios más completos que se hayan hecho hasta esa fecha sobre diferencias culturales entre países. La investigación tuvo gran impacto en muchos ámbitos de la sociología y la administración, especialmente en negociación, gestión de equipos multiculturales y en la configuración de estrategias de marketing internacional (Hofstede, 2001; Hidalgo et al., 2007; Manzur et al., 2012). Hofstede (1980) analizó 70 países y simplificó complejos patrones culturales de conducta en sólo cuatro dimensiones culturales (aunque actualmente Hofstede utiliza seis dimensiones culturales). El trabajo de Hofstede ha mostrado que hay agrupamientos culturales a nivel nacional que afectan el comportamiento de las sociedades y las organizaciones.

De acuerdo con Hofstede (1991: 4), la cultura es siempre un fenómeno colectivo, debido a que es compartida con personas que viven o vivieron en un mismo ambiente social, en la cual la cultura es aprendida. La cultura se aprende, no se hereda. Ésta se deriva de un ambiente social, no de los genes. La cultura puede ser distinguida de la naturaleza humana, por un lado, y de la personalidad de los individuos, por otro (véase Figura $1^{1}$ ). Hofstede (1991: 5) define "cultura" como la programación mental colectiva que distingue a miembros de un grupo o categoría de gente de los de otros.

Hofstede (2001: 2) señala que los programas mentales pueden ser heredados (transferidos a través de nuestros genes) o pueden ser aprendidos después de nacer; asimismo, define tres niveles de programación mental: individual, colectiva y universal. Hofstede indica que a nivel individual, al menos una parte de la programación debe ser heredada; de lo contrario, es difícil explicar diferencias en habilidades y temperamento entre niños de una misma familia y ambiente. A nivel colectivo, Hofstede (2001: 3) señala que la mayoría de nuestra programación mental es aprendida; lo ejemplifica con los estadounidenses, quienes son una multitud de variaciones genéticas, pero que, sin embargo, muestran una programación mental colectiva que es percibida por los extranjeros. Hofstede (2001: 3) indica que el nivel universal de la programación mental es compartido por todos los seres humanos y se refiere a la naturaleza humana. Ésta es heredada por medio de los genes, deter-

1 Todas las figuras y tablas se encuentran en el Anexo, al final del presente artículo (Nota del Editor). 
minando nuestro funcionamiento físico y psicológico básico. La capacidad humana de sentir miedo, furia, amor, placer, enojo, etcétera pertenecen a este nivel de programación mental.

Hofstede (1991: 5) se refiere a grupo como el conjunto de personas que mantienen contacto unas con otras. Una categoría de gente consiste en personas que sin necesariamente tener contacto entre ellas, tienen algo en común. Por ejemplo, todas las mujeres ejecutivas, deportistas, vegetarianos, filósofos, escritores, etc. Esta definición de cultura se refiere más tangiblemente a considerar las características personales que sean comunes y estándares en una sociedad dada. Ya que existe una gran variedad de personalidades individuales en cualquier sociedad, la que se observa con mayor frecuencia (en términos estadísticos) ha sido usada para aproximarse a la cultura nacional (Clark, 1990; Nakata y Sivakumar, 1996). El término “cultura”, en este sentido, puede ser aplicado a naciones, organizaciones, ocupaciones y profesiones, grupos religiosos, grupos étnicos, etcétera.

El concepto de cultura es más aplicable a sociedades que a naciones. Sin embargo, históricamente, muchas naciones han desarrollado una forma conjunta, aún si ello consiste en grupos claramente diferentes y aún si éstas contienen minorías menos integradas. Además, también hay "fuerzas" que dificultan la integración. Por ejemplo, existen grupos religiosos, étnicos que buscan su propia identidad. Empero, al interior de las naciones han existido a través del tiempo "fuerzas" que posibilitan la integración: un lenguaje nacional dominante, medios de comunicación masivos comunes, sistema educacional nacional, ejército nacional, sistema político nacional, representación nacional en eventos deportivos, mercados nacionales de productos y servicios, etcétera.

De acuerdo con Hofstede (2001: 12), los orígenes de las distancias culturales de una nación a otra se encuentran arraigados en la historia universal; señala que en algunos casos son posibles las explicaciones de las causas de las distancias culturales. En muchos otros casos uno podría simplemente asumir que pequeñas diferencias hace cientos y miles de años atrás, transferidas de generación en generación, llevaron a esas pequeñas diferencias culturales a crecer y crecer hasta llegar a las distancias culturales actuales entre países.

\section{El estudio de House et al. (2004)}

House et al. (2004: 12), incorporando las dimensiones culturales de Hofstede $(1980 ; 2001)$ y utilizando métodos cualitativos, identificaron y 
midieron nueve dimensiones culturales para 62 países. El trabajo de House et al. (2004) también es conocido como el estudio o proyecto GLOBE (Global Leadership and Organizational Behavior Effectiveness Research). En este artículo, con el objetivo de presentar una metodología para medir cuantitativamente y representar gráficamente las distancias culturales entre los países latinoamericanos, se utilizan los puntajes de los países latinoamericanos en las nueve dimensiones culturales medidas por House et al. (2004). En los diez países latinoamericanos incluidos en el estudio de House et al. (2004) se encuestaron a 1.527 gerentes de nivel medio (ni presidentes ni vicepresidentes, y dos niveles por encima de los trabajadores) de empresas de tres sectores empresariales: telecomunicaciones, finanzas e industria procesadora de alimentos.

Los diez países latinoamericanos incluidos y el total de encuestas efectuadas por House et al. (2004) son los siguientes: Argentina (153), Bolivia (99), Brasil (265), Colombia (302), Costa Rica (114), Ecuador (49), El Salvador (25), Guatemala (110), México (260) y Venezuela (150). House et al. (2004: 11) midieron nueve dimensiones de la cultura nacional a través de las respuestas de los gerentes latinoamericanos sobre cómo es su cultura y cómo querían ellos que fuese su cultura en cada una de las nueve dimensiones culturales (véase Tabla 1).

La utilización de una muestra relativamente homogénea (i.e., gerentes de nivel medio de tres sectores empresariales) al interior de los países permite capturar las dimensiones culturales de una manera más limpia en comparación a usar una mayor heterogeneidad en las muestras al interior de cada uno de los países. Las dimensiones culturales medidas demostraron ser unidimensionales, con coeficientes alfa de Cronbach adecuados y con una fuerte capacidad de agregación social (House et al., 2004).

\section{Las dimensiones culturales medidas por House et al. (2004)}

El estudio de House et al. (2004: 11) midió nueve dimensiones de la cultura nacional: asertividad, colectivismo, colectivismo familiar, distancia de poder, evitar la incertidumbre, igualdad de género, orientación al desempeño, orientación al futuro y orientación humana. A continuación se describen brevemente estas nueve dimensiones culturales:

Asertividad. House et al. (2004) distinguieron entre sociedades donde la gente es muy agresiva, afirmativa, dura en sus opiniones, dominante y recurre a la fuerza física para dirimir sus diferencias, de otras de comporta- 
mientos menos agresivos. Para los valores tradicionales del mundo esto tiene que ver con culturas masculinas y culturas femeninas (Hofstede, 1980): el ser tierno, poco dominante, suave, poco agresivo, se identifica con el temperamento "femenino", y los comportamientos más agresivos, dominantes, de predominancia física, opiniones fuertes, se identifican con el temperamento "masculino".

Colectivismo. Se refiere al grado en el cual una sociedad valora la lealtad al grupo, el compromiso con las normas grupales y actividades colectivas, cohesividad social e intensa sociabilización por sobre los objetivos personales, autonomía y privacidad de las personas (Hofstede, 2001; Triandis, 2004).

Colectivismo familiar. Esta dimensión trata de identificar la orientación hacia valores colectivos en la relación entre padres e hijos y la importancia de la familia en la sociedad.

Distancia de poder. Esta dimensión se define como el grado en el cual una sociedad acepta la distribución desigual de poder en las instituciones y las organizaciones (De Mooij y Hofstede, 2002; Hodgetts y Luthans, 1993; Ryan et al., 1999). Las "instituciones" son los elementos básicos de la sociedad tales como la familia, la escuela y la comunidad. Las "organizaciones" son grupos sociales compuestos por personas, tareas y administración que forman una estructura sistemática de relaciones de interacción, tendientes a producir bienes o servicios o normativas para satisfacer las necesidades de una comunidad dentro de un entorno, y así poder lograr el propósito distintivo que es su misión (Hofstede, 2001).

Evitar la incertidumbre. Es el grado en el cual los miembros de la sociedad se sienten inconfortables en situaciones no estructuradas (Hofstede, 2001). Hofstede (1991) señala que esta dimensión también puede ser definida como el grado en el cual las personas en un país prefieren situaciones estructuradas sobre situaciones no estructuradas. Una sociedad orientada a reducir la incertidumbre instruye normas, leyes, regulaciones y controles para disminuir la cantidad de incertidumbre (De Mooij y Hofstede, 2002; Lu et al., 1999; Shane, 1995). Estas reglas pueden ser escritas, pero también pueden ser no escritas e impuestas por tradición (Stohl, 1993). En estas culturas la gente busca situaciones estructuradas, conocer con precisión qué va a ocurrir, y, por lo tanto, la predicción de los eventos es muy valorado (Ryan et al., 1999; Triandis, 2004).

Igualdad de género. Esta dimensión cultural trata de identificar hasta qué punto la gente de un país prefiere minimizar las diferencias de roles y estatus entre hombres y mujeres. 
Orientación al desempeño. Se trata de identificar el grado en el cual las personas están orientadas a la excelencia, al mejoramiento continuo, a obtener desempeño sobresaliente y al logro de los resultados.

Orientación al futuro. Esta dimensión cultural trata de identificar si los individuos están orientados hacia el futuro, o si los criterios y valores están centrados en el presente o en el pasado.

Orientación humana. Esta dimensión intenta determinar el grado en el cual los valores culturales de un país apoyan y recompensan a la gente por ser altruistas, justos, compasivos, amistosos y sensibles con los demás.

\section{Estudio}

\section{Medición cuantitativa de las distancias culturales}

Con el objetivo de medir cuantitativamente las distancias culturales entre los diez países latinoamericanos incluidos en House et al. (2004), en el presente estudio se usa la metodología propuesta por Kogut y Singh (1988), quienes desarrollaron un índice de distancia cultural para las dimensiones culturales medidas por Hofstede (1980). Kogut y Singh (1988) combinaron las dimensiones culturales de Hofstede (1980) en una medida agregada de distancias culturales entre países. Dicha medida ha sido ampliamente utilizada en posteriores investigaciones en diversas áreas de las ciencias sociales (e.g., Agarwal, 1993; Barkema et al., 1996; Roth y O’Donnel, 1996). Este índice se forma a partir del promedio de las desviaciones de los índices (I) entre el país "X" y el país " $Y$ " en cada una de las "i" dimensiones culturales, corregidas por la varianza (V) de cada "i” dimensión cultural (Kogut y Singh, 1988: 422). Algebraicamente, el índice de "distancia cultural" entre el país "X" y el país "Y" (Cultural Distance - CDxy) para el estudio de House et al. (2004), de nueve dimensiones culturales, se puede calcular con la siguiente ecuación 1 :

$$
C D_{x y}=\left\{\sum_{i=1}^{9} \frac{\left(I_{i y}-I_{i x}\right)^{2}}{V_{i}}\right\} / 9
$$

La Tabla 2 muestra las matrices de distancias culturales calculadas en este estudio para los diez países latinoamericanos incluidos en el trabajo de House et al. (2004). Se presentan las distancias culturales que los diez países 
latinoamericanos poseen actualmente (matriz de distancias culturales actuales) y también las distancias culturales que potencialmente podrían tener en el futuro los diez países latinoamericanos (matriz de distancias culturales potenciales).

\section{Distanciamiento-acercamiento de las culturas nacionales}

El diferencial entre cómo vive la gente y cómo desea vivir es un factor de insatisfacción, y por lo tanto, de cambio cultural. Dada la diferencia entre cómo vive la gente (matriz de distancias culturales actuales) y cómo desea vivir (matriz de distancias culturales potenciales) en América Latina, los esfuerzos por disminuir ese diferencial serán una tendencia importante de cambios culturales en el futuro (House et al., 2004). La Tabla 3 indica el porcentaje de incremento potencial de la distancia cultural entre los diez países latinoamericanos. Valores positivos (negativos) indican que existe un potencial distanciamiento (acercamiento) cultural entre los países. Por ejemplo, Brasil y El Salvador muestran un relevante potencial de distanciamiento cultural (la distancia cultural se incrementaría en un 421,3\%), dado que brasileños y salvadoreños difieren en la cultura nacional que desean tener en el futuro. Por otra parte, las culturas nacionales de Ecuador y Venezuela presentan un significativo potencial de acercamiento en las próximas décadas (la distancia cultural se reduciría en un $85,1 \%$ ). Consecuentemente, para cada uno de los diez países latinoamericanos se puede proyectar qué culturas nacionales se acercarán o distanciarán en el futuro.

\section{Representación gráfica de las distancias culturales}

Utilizando como variables de entrada las distancias culturales calculadas entre los diez países latinoamericanos (matrices de distancias culturales actuales y potenciales; véase Tabla 2), se realizaron dos escalamientos multidimensionales (EMD) con el objetivo de representar gráficamente estas distancias culturales en mapas. La Figura 2 (EMD; Stress = ,09417; RSQ = ,95240; índices de ajuste aceptables, Malhotra, 2004; Hair et al., 2006) presenta el mapa de las distancias culturales actuales encontradas entre los diez países latinoamericanos, usando los datos de la matriz de distancias culturales actuales.

El Stress mide el mal ajuste o la proporción de la varianza de los datos de escala óptima que no explica el EMD. Un Stress del 0,20 o superior es 
considerado malo, 0,05 es considerado bueno y 0,025 es visto como excelente (Malhotra, 2004: 617-618). RSQ (R cuadrado) señala la proporción de varianza de los datos de escala óptima que pueden explicarse con el EMD. Se considera aceptable valores de 0,60 en adelante (Malhotra, 2004: 617). La Figura 3 (EMD; Stress = ,06359; RSQ = ,97679; índices de ajuste aceptables, Malhotra, 2004; Hair et al., 2006) presenta el mapa de las distancias culturales potenciales encontradas entre los diez países latinoamericanos, ocupando los datos de la matriz de distancias culturales potenciales.

En ambos mapas de distancias culturales se puede observar que Brasil muestra una pequeña distancia cultural con Argentina y Colombia. Por otro lado, Brasil presenta una gran distancia cultural con México y Ecuador. En ambos mapas se puede observar que México presenta una pequeña distancia cultural con Ecuador. En contraste, México exhibe una gran distancia cultural con Argentina y Brasil (véanse Figuras 2 y 3 ).

\section{Interpretación de las dimensiones de los mapas de distancias culturales}

Posteriormente, se siguió la metodología sugerida por Fernández et al. (2003) para interpretar las dimensiones de los mapas obtenidos en los EMD. Como consecuencia, se realizó una regresión lineal para cada una de las nueve dimensiones culturales, es decir, la variable dependiente es el valor del índice (I) de la "i" dimensión cultural para cada país " $\mathrm{X}$ " y las variables independientes son las coordenadas del EMD (Dim1, Dim2) para cada país "X” (véase ecuación 2). De acuerdo con Fernández et al. (2003), la condición para una interpretación satisfactoria es que los coeficientes (betas) sean significativos estadísticamente (valor-p $<0,01$ ) y un $\mathrm{R}$ cuadrado de 0,70 es considerado aceptable.

$$
I_{i x}=\alpha+\beta 1^{*} \operatorname{Dim} 1_{x}+\beta 1^{*} \operatorname{Dim} 2_{x}+\varepsilon_{x}
$$

Para el EMD distancias culturales actuales, los resultados de la Tabla 4 indican con significación estadística que la dimensión 1 es explicada principalmente por las variables de colectivismo (negativamente) y distancia de poder, y la dimensión 2 se explica, sobre todo, por la distancia de poder (negativamente). Luego, es posible observar que la actual distancia cultural que presentan Costa Rica y Bolivia del resto de los países latinoamericanos se puede explicar tanto por la baja distancia de poder como por el alto colectivismo que muestran en relación con el resto los países. 
Para el EMD distancias culturales potenciales, los resultados de la Tabla 5 indican con significación estadística que la dimensión 1 es explicada principalmente por la orientación humana, orientación al futuro y orientación al desempeño, y la dimensión 2 se explica, sobre todo, por la orientación humana y la igualdad de género. Se consideró aceptables los ajustes para orientación al futuro (valor-p $=0,012)$ e igualdad de género $(\mathrm{R}$ cuadrado $=0,697)$ al observarse en estos dos casos valores muy cercanos a los valores aceptables (valor-p < 0,01 y R cuadrado > 0,7), de acuerdo con Fernández et al. (2003). Entonces es posible observar que los individuos de los países de Argentina, Brasil y Colombia presentan un mayor deseo que el resto de los países latinoamericanos por tener una orientación más humana y una mayor igualdad de género, lo cual llevaría a éstos a un potencial distanciamiento cultural del resto de las naciones latinoamericanas en el futuro.

\section{Análisis del caso de México}

En esta sección se analiza el caso de México para ilustrar las implicancias de este estudio para los diez países latinoamericanos. La Figura 4 expone las distancias culturales actuales y potenciales que tiene México con los otros nueve países latinoamericanos. El presente trabajo muestra que hoy en día México exhibe una gran distancia cultural con Argentina, Guatemala y El Salvador. En contraste, México presenta en la actualidad una pequeña distancia cultural con Costa Rica y Ecuador. Además, es posible observar que México muestra un potencial distanciamiento cultural con Brasil (la distancia cultural se incrementaría en un $66,2 \%$ ) y un potencial acercamiento cultural con Guatemala (la distancia cultural se reduciría en un $76,3 \%$ ).

Las distancias culturales actuales y potenciales con otros países tienen implicancias en el desarrollo de América Latina y de México, en particular. Por ejemplo, se ha observado que la distancia cultural puede influir sobre el intercambio comercial entre países (Lee, 1998). Culturas más similares pueden generar acuerdos, relaciones, comportamientos comunes, facilitando el intercambio comercial entre los países. Utilizando datos de SE (2015), se analizó la asociación entre la distancia cultural actual y el crecimiento en las exportaciones de México hacia los nueve países latinoamericanos en los últimos diez años (2004-2014).

La Figura 5 muestra que los países que tienen actualmente una menor distancia cultural con México (e.g., Brasil, Colombia) presentan un mayor crecimiento en las exportaciones de México en los últimos diez años. En contraste, los países con mayor distancia cultural (e.g., Argentina, Guatemala, El 
Salvador, Venezuela) registran un menor crecimiento en las exportaciones de México en los últimos diez años.

Una menor distancia cultural entre países también puede facilitar la inmigración entre éstos (Tadesse y White, 2010). Con datos de INEDI (2015) se analizó la asociación entre la distancia cultural actual y el crecimiento en el periodo 1970-2010 en el número de inmigrantes que recibió México provenientes de los nueve países latinoamericanos. La Figura 6 muestra que los países que tienen actualmente una menor distancia cultural con México (e.g., Colombia) presentan un mayor crecimiento en el número de inmigrantes que recibió México en el periodo 1970-2010. En contraste, los países con mayor distancia cultural con México (e.g., Guatemala) presentan un menor crecimiento en el número de inmigrantes que recibió México en el periodo 1970-2010.

Debido a que la cultura también influye sobre los grupos de investigación (Kedia et al., 1992), los cuales se caracterizan por tener un horizonte de largo plazo al momento de trabajar y definir líneas de investigación, la distancia cultural potencial entre los países puede influir sobre el número de copublicaciones científicas entre grupos de investigación de distintas naciones. Grupos de investigación de países con una mayor distancia cultural potencial pueden ser más atractivos dadas las diferencias culturales potenciales para los grupos de investigación de un país determinado, debido a las diferencias entre investigadores, unidades de prueba, contextos, etcétera.

Con datos de Lozano et al. (2006) se analizó la asociación entre la distancia cultural potencial y el número de copublicaciones científicas entre grupos de investigación de México con grupos de investigación de los otros nueve países latinoamericanos. La Figura 7 muestra que los países con una mayor distancia cultural potencial con México (e.g., Brasil) presentan un mayor número de copublicaciones científicas con México. En contraste, los países con una menor distancia cultural potencial con México (e.g., Bolivia, Guatemala) exhiben un menor número de copublicaciones científicas con México.

Es importante señalar que en esta sección se describen asociaciones entre variables para ilustrar las implicancias del presente estudio. Futuras investigaciones en América Latina pueden incorporar variables de control que permitan concluir causalidad entre las variables analizadas u otras variables que el investigador quisiera examinar. 


\section{Conclusiones e implicancias}

Esta investigación aborda la medición cuantitativa y representación gráfica de las distancias culturales entre diez países latinoamericanos (Argentina, Bolivia, Brasil, Colombia, Costa Rica, Ecuador, El Salvador, Guatemala, México y Venezuela), utilizando las nueve dimensiones culturales medidas por House et al. (2004) para estos diez países latinoamericanos y empleando las metodologías sugeridas por Kogut y Singh (1988) y Fernández et al. (2003), para medir cuantitativamente, representar gráficamente e interpretar las distancias culturales entre países en América Latina.

Este estudio permitirá trabajar cuantitativamente la variable distancia cultural entre los países latinoamericanos, la que tradicionalmente muchas investigaciones a nivel mundial y en América Latina han abordado cualitativamente (para una revisión de éstos véase Alasuutari, 1995). Esto posibilitará dar un nuevo enfoque a futuras investigaciones en América Latina que incorporen la distancia cultural entre países en sus análisis. Por lo tanto, las implicancias de este trabajo son muchas para los investigadores en las distintas áreas de las ciencias sociales: se ha presentado una metodología para medir cuantitativamente y representar gráficamente distancias culturales y proyectar posibles cambios en las distancias culturales entre los diez países latinoamericanos incluidos en House et al. (2004).

Los investigadores en una amplia diversidad de áreas de las ciencias sociales pueden utilizar las distancias culturales calculadas como variables de entrada en modelos que incorporen este concepto, ya sea como variable dependiente, variable independiente o variable de control en futuras investigaciones en América Latina. La medición cuantitativa y representación gráfica de las distancias culturales entre los diez países latinoamericanos no sólo se podrá usar en enfoques cuantitativos, sino también en enfoques cualitativos, al poder ocupar los resultados de este estudio como datos secundarios para la elaboración de preguntas de investigación, comprender los resultados de una investigación, etcétera.

Los reguladores, directivos y administradores también pueden emplear esta herramienta para una toma de decisiones sensibilizada por las distancias culturales entre los países latinoamericanos. Es de esperar que en el futuro tanto investigadores como tomadores de decisión puedan contar con estudios que, utilizando metodologías comparables, incorporen en su análisis un mayor número de países latinoamericanos (i.e., no sólo los incluidos por House et al., 2004), para poder evaluar la evolución de las distancias culturales entre países y su potencial impacto en las diversas áreas de las ciencias sociales. 
Pablo Farías. Medición y representación gráfica de las distancias culturales entre paises latinoamericanos

\section{Bibliografía}

Alasuutari, Pertti (1995), Researching culture: Qualitative method and cultural studies, London: Sage.

Agarwal, Sanjeev (1993), "Influence of formalization on role stress, organizational commitment, and work alienation of salespersons: A cross-national comparative study", en Journal of International Business Studies, vol. 24, núm. 4, England.

Barkema, Harry et al. (1996), "Foreign Entry, Cultural Barriers and Learning”, en Strategic Management Journal, vol. 17, núm. 1, Holland.

Clark, Terry (1990), "International Marketing and National Character: A review and proposal for an integrative theory”, en Journal of Marketing, vol. 54, núm. 4, USA.

De Mooij, Marieke y Geert Hofstede (2002), "Convergence and divergence in consumer behaviour: implications for international retailing”, en Journal of Retailing, vol. 78, núm. 1 , USA.

Farías, Pablo (2015), "Determinants of the success of global and local brands in Latin America”, en RAE, vol. 55, núm. 5, Brazil.

Fernández, Pedro et al. (2003), "Mapas Perceptuales: Interpretación de Ejes del MDS Usando Regresión”, en Documento de trabajo, Chile: Universidad Técnica Federico Santa María.

Hair, Joseph et al. (2006), Multivariate data analysis, Upper Saddle River, NJ: Pearson Prentice Hall.

Hidalgo, Pedro et al. (2007), "La cultura nacional y su impacto en los negocios: El caso chileno”, en Estudios Gerenciales, vol. 23, núm. 105, Colombia.

Hodgetts, Richard y Fred Luthans (1993), “U.S. multinationals' compensation strategies for local management: Cross-cultural implications", en Compensation \& Benefits Review, vol. 25, núm. 2, USA: California.

Hofstede, Geert (1980), Culture's Consequences: International Differences in work-related values, Beverly Hills, USA, CA: Sage.

Hofstede, Geert (1991), Cultures and Organizations: Software of the Mind, USA, CA: McGraw-Hill.

Hofstede, Geert (2001), Culture's Consequences: Comparing Values, Behaviors, Institutions, and Organizations across Nations, USA, CA: Sage.

House, Robert J. et al. (2004). Culture, leadership, and organizations: The GLOBE study of 62 societies, USA, CA: Sage.

Kedia, Ben, Robert Keller y Scott D. Jullan (1992), "Dimensions of national culture and the productivity of R\&D units", en The Journal of High Technology Management Research, vol. 3, núm. 1, Holland.

Kogut, Bruce y Harbir Singh (1988), “The effect of national culture on the choice of entry mode”, en Journal of International Business Studies, vol. 19, núm. 3, England.

Lee, Dong-Jin (1998), “The effect of cultural distance on the relational exchange between exporters and importers: the case of Australian exporters", en Journal of Global Marketing, vol. 11, núm. 4, England. 
Lozano, Rosa et al. (2006), "Indicadores de colaboración científica inter-centros en los países de América Latina”, en Interciencia: Revista de Ciencia y Tecnología de América, vol. 31, núm. 4, Venezuela.

Lu, Long-Chuan, Gregory M. Rose y Jeffrey G. Blodgett (1999), "The effects of cultural dimensions on ethical decision making in marketing: An exploratory study", en Journal of Business Ethics, vol. 18, núm. 1, Germany.

Malhotra, Naresh K. (2004), Investigación de Mercados. Un Enfoque Aplicado, México: Pearson Educación.

Manzur, Enrique et al. (2012), "Comparative Advertising Effectiveness in Latin America: Evidence from Chile”, en International Marketing Review, vol. 29, núm. 3, England.

Meunier, Isabel y Marcelo de Almeida Medeiros (2013), "Construindo a América do Sul: identidades e interesses na formação discursiva da Unasul”, en Dados, vol. 56, núm. 3, Brazil.

Nakata, Cheryl y Kumar Sivakumar (1996), "National culture and new product development: An integrative review”, en Journal of Marketing, vol. 60, núm. 1, USA.

Olavarrieta, Sergio et al. (2013), "Store Price Promotion Strategies, Price Perceptions, Search and Purchase Intentions", en Academia Revista Latinoamericana de Administración, vol. 26, núm. 3, Colombia.

Roth, Kendall y Sharon O’Donnel (1996), “Foreign Subsidiary Compensation Strategy: An Agency Theory Perspective”, en Academy of Management Journal, vol. 39, núm. 3, USA.

Ryan, Ann Marie et al. (1999), "An international look at selection practices: Nation and culture as explanations for variability in practice”, en Personnel Psychology, vol. 52, núm. 2, USA.

Shane, Scott (1995), "Uncertainty avoidance and the preference for innovation championing roles”, en Journal of International Business Studies, vol. 26, núm. 1, England.

Shenkar, Oded (2001), "Cultural Distance Revisited: Towards a More Rigorous Conceptualization and Measurement of Cultural Differences", en Journal of International Business Studies, vol. 32, núm. 3, England.

Stohl, Cynthia (1993), "European managers' interpretations of participation: A semantic network analysis”, en Human Communication Research, vol. 21, núm. 1, USA.

Tadesse, Bedassa y Roger White (2010), "Cultural distance as a determinant of bilateral trade flows: do immigrants counter the effect of cultural differences?", en Applied Economics Letters, vol. 17, núm. 2, England.

Triandis, Harry C. (2004), “The many dimensions of culture”, en Academy of management executive, vol. 18 , núm. 1 , USA.

\section{Recursos electrónicos}

INEDI (2015), “Nacidos en otro país”. Disponible en http://www.inegi.org.mx/ inegi/contenidos/espanol/prensa/contenidos/Articulos/sociodemograficas/ nacidosenotropais.pdf [30 de agosto de 2015].

SE (2015), "Información Estadística y Arancelaria”. Disponible en http://www.economia. gob.mx/comunidad-negocios/comercio-exterior/informacion-estadistica-y-arancelaria [1 de septiembre de 2015]. 
Pablo Farías. Medición y representación gráfica de las distancias culturales entre países latinoamericanos

\section{Anexo}

\section{Tabla 1}

Dimensiones culturales medidas por House et al. (2004)

\begin{tabular}{lcccccccccc}
\hline \multicolumn{10}{c}{ Cultura actual: ¿Cómo es la sociedad? } \\
\hline & ARG & BOL & BRA & COL & CR & ECU & SAL & GUA & MEX & VEN \\
\hline Asertividad & 3,70 & 4,12 & 3,70 & 3,08 & 4,37 & 3,73 & 2,87 & 3,76 & 3,58 & 3,32 \\
\hline Colectivismo & 3,66 & 4,04 & 3,83 & 3,81 & 3,93 & 3,90 & 3,71 & 3,70 & 4,06 & 3,96 \\
\hline $\begin{array}{l}\text { Colectivismo } \\
\text { familiar }\end{array}$ & 5,51 & 5,47 & 5,18 & 5,73 & 5,32 & 5,81 & 5,35 & 5,63 & 5,71 & 5,53 \\
\hline $\begin{array}{l}\text { Distancia de } \\
\text { poder }\end{array}$ & 5,64 & 4,51 & 5,33 & 5,56 & 4,74 & 5,60 & 5,68 & 5,60 & 5,22 & 5,40 \\
\hline $\begin{array}{l}\text { Evitar la in- } \\
\text { certidumbre }\end{array}$ & 3,65 & 3,35 & 3,60 & 3,57 & 3,82 & 3,68 & 3,62 & 3,30 & 4,18 & 3,44 \\
\hline $\begin{array}{l}\text { Igualdad de } \\
\text { género }\end{array}$ & 3,49 & 3,55 & 3,31 & 3,67 & 3,56 & 3,07 & 3,16 & 3,02 & 3,64 & 3,62 \\
\hline $\begin{array}{l}\text { Orientación } \\
\text { al desempeño }\end{array}$ & 3,65 & 3,61 & 4,04 & 3,94 & 4,12 & 4,20 & 3,72 & 3,81 & 4,10 & 3,32 \\
\hline $\begin{array}{l}\text { Orientación } \\
\text { al futuro }\end{array}$ & 3,08 & 3,61 & 3,81 & 3,27 & 3,60 & 3,74 & 3,80 & 3,24 & 3,87 & 3,35 \\
\hline $\begin{array}{l}\text { Orientación } \\
\text { humana }\end{array}$ & 3,99 & 4,05 & 3,66 & 3,72 & 4,39 & 4,65 & 3,71 & 3,89 & 3,98 & 4,25 \\
\hline
\end{tabular}


Convergencia Revista de Ciencias Sociales, núm. 70, 2016, Universidad Autónoma del Estado de México

Cultura potencial: ¿Cómo debe ser la sociedad?

\begin{tabular}{lcccccccccc}
\hline & ARG & BOL & BRA & COL & CR & ECU & SAL & GUA & MEX & VEN \\
\hline Asertividad & 4,22 & 3,58 & 4,22 & 3,59 & 3,25 & 3,32 & 2,97 & 3,43 & 3,46 & 3,70 \\
\hline Colectivismo & 5,32 & 5,10 & 5,62 & 5,38 & 5,18 & 5,41 & 5,65 & 5,23 & 4,92 & 5,39 \\
\hline $\begin{array}{l}\text { Colectivismo } \\
\text { familiar }\end{array}$ & 6,15 & 6,00 & 5,15 & 6,25 & 6,08 & 6,17 & 6,52 & 6,14 & 5,95 & 6,17 \\
\hline $\begin{array}{l}\text { Distancia de } \\
\text { poder }\end{array}$ & 2,33 & 3,41 & 2,35 & 2,04 & 2,58 & 2,30 & 2,68 & 2,35 & 2,85 & 2,29 \\
\hline $\begin{array}{l}\text { Evitar la in- } \\
\text { certidumbre }\end{array}$ & 4,66 & 4,70 & 4,99 & 4,98 & 4,58 & 5,16 & 5,32 & 4,88 & 5,26 & 5,26 \\
\hline $\begin{array}{l}\text { Igualdad de } \\
\text { género }\end{array}$ & 4,98 & 4,75 & 4,99 & 5,00 & 4,64 & 4,59 & 4,66 & 4,53 & 4,73 & 4,82 \\
\hline $\begin{array}{l}\text { Orientación } \\
\text { al desempeño }\end{array}$ & 6,35 & 6,05 & 6,13 & 6,42 & 5,90 & 6,32 & 6,58 & 6,14 & 6,16 & 6,35 \\
\hline $\begin{array}{l}\text { Orientación } \\
\text { al futuro }\end{array}$ & 5,78 & 5,63 & 5,69 & 5,68 & 5,20 & 5,94 & 5,98 & 5,91 & 5,86 & 5,79 \\
\hline $\begin{array}{l}\text { Orientación } \\
\text { humana }\end{array}$ & 5,58 & 5,07 & 5,68 & 5,61 & 4,99 & 5,26 & 5,46 & 5,26 & 5,10 & 5,31 \\
\hline
\end{tabular}

Fuente: House et al. (2004). 
Pablo Farías. Medición y representación gráfica de las distancias culturales entre paises latinoamericanos

\section{Tabla 2}

\section{Distancias culturales actuales y potenciales}

\begin{tabular}{lcccccccccc}
\hline \multicolumn{1}{c}{ Matriz de distancias culturales actuales } \\
\hline CDxy & ARG & BOL & BRA & COL & CR & ECU & SAL & GUA & MEX & VEN \\
\hline Argentina & - & 2,348 & 1,677 & 0,796 & 2,240 & 2,409 & 1,490 & 0,750 & 2,832 & 1,060 \\
\hline Bolivia & 2,348 & - & 1,736 & 2,385 & 1,086 & 2,848 & 3,048 & 2,390 & 2,339 & 1,227 \\
\hline Brasil & 1,677 & 1,736 & - & 1,750 & 1,445 & 2,392 & 0,816 & 1,605 & 1,992 & 2,151 \\
\hline Colombia & 0,796 & 2,385 & 1,750 & - & 2,746 & 2,317 & 1,427 & 1,301 & 1,840 & 1,182 \\
\hline Costa Rica & 2,240 & 1,086 & 1,445 & 2,746 & - & 1,980 & 3,273 & 2,867 & 1,542 & 2,323 \\
\hline Ecuador & 2,409 & 2,848 & 2,392 & 2,317 & 1,980 & - & 2,511 & 1,767 & 1,833 & 2,500 \\
\hline El Salvador & 1,490 & 3,048 & 0,816 & 1,427 & 3,273 & 2,511 & - & 1,365 & 2,721 & 1,872 \\
\hline Guatemala & 0,750 & 2,390 & 1,605 & 1,301 & 2,867 & 1,767 & 1,365 & - & 3,599 & 1,723 \\
\hline México & 2,832 & 2,339 & 1,992 & 1,840 & 1,542 & 1,833 & 2,721 & 3,599 & - & 2,499 \\
\hline Venezuela & 1,060 & 1,227 & 2,151 & 1,182 & 2,323 & 2,500 & 1,872 & 1,723 & 2,499 & - \\
\hline & & Matriz & de distancias culturales & potenciales & & & \\
\hline CDxy & ARG & BOL & BRA & COL & CR & ECU & SAL & GUA & MEX & VEN \\
\hline Argentina & - & 2,270 & 1,422 & 0,554 & 3,162 & 1,790 & 2,862 & 1,640 & 2,330 & 0,986 \\
\hline Bolivia & 2,270 & - & 3,430 & 2,906 & 1,153 & 2,096 & 3,522 & 1,392 & 0,947 & 2,060 \\
\hline Brasil & 1,422 & 3,430 & - & 1,793 & 4,188 & 2,803 & 4,256 & 2,891 & 3,310 & 1,866 \\
\hline Colombia & 0,554 & 2,906 & 1,793 & - & 3,127 & 1,193 & 1,717 & 1,554 & 2,188 & 0,512 \\
\hline Costa Rica & 3,162 & 1,153 & 4,188 & 3,127 & - & 2,543 & 4,607 & 1,661 & 2,155 & 2,660 \\
\hline Ecuador & 1,790 & 2,096 & 2,803 & 1,193 & 2,543 & - & 0,754 & 0,308 & 1,034 & 0,371 \\
\hline El Salvador & 2,862 & 3,522 & 4,256 & 1,717 & 4,607 & 0,754 & - & 1,732 & 2,451 & 1,116 \\
\hline Guatemala & 1,640 & 1,392 & 2,891 & 1,554 & 1,661 & 0,308 & 1,732 & - & 0,854 & 0,804 \\
\hline México & 2,330 & 0,947 & 3,310 & 2,188 & 2,155 & 1,034 & 2,451 & 0,854 & - & 1,029 \\
\hline Venezuela & 0,986 & 2,060 & 1,866 & 0,512 & 2,660 & 0,371 & 1,116 & 0,804 & 1,029 & - \\
\hline Eunte: Elab & & & & & & & & & & \\
\hline
\end{tabular}

Fuente: Elaboración propia. 


\section{Tabla 3}

Potencial crecimiento de la distancia cultural

\begin{tabular}{lcccccccccc}
\hline & ARG & BOL & BRA & COL & CR & ECU & SAL & GUA & MEX & VEN \\
\hline Argentina & - & $-3,3 \%$ & $-15,2 \%$ & $-30,4 \%$ & $41,2 \%$ & $-25,7 \%$ & $92,1 \%$ & $118,7 \%$ & $-17,7 \%$ & $-7,0 \%$ \\
\hline Bolivia & $-3,3 \%$ & - & $97,6 \%$ & $21,8 \%$ & $6,2 \%$ & $-26,4 \%$ & $15,6 \%$ & $-41,7 \%$ & $-59,5 \%$ & $67,9 \%$ \\
\hline Brasil & $-15,2 \%$ & $97,6 \%$ & - & $2,5 \%$ & $189,8 \%$ & $17,2 \%$ & $421,3 \%$ & $80,1 \%$ & $66,2 \%$ & $-13,2 \%$ \\
\hline Colombia & $-30,4 \%$ & $21,8 \%$ & $2,5 \%$ & - & $13,9 \%$ & $-48,5 \%$ & $20,3 \%$ & $19,4 \%$ & $18,9 \%$ & $-56,7 \%$ \\
\hline Costa Rica & $41,2 \%$ & $6,2 \%$ & $189,8 \%$ & $13,9 \%$ & - & $28,4 \%$ & $40,8 \%$ & $-42,1 \%$ & $39,7 \%$ & $14,5 \%$ \\
\hline Ecuador & $-25,7 \%$ & $-26,4 \%$ & $17,2 \%$ & $-48,5 \%$ & $28,4 \%$ & - & $-70,0 \%$ & $-82,6 \%$ & $-43,6 \%$ & $-85,1 \%$ \\
\hline El Salvador & $92,1 \%$ & $15,6 \%$ & $421,3 \%$ & $20,3 \%$ & $40,8 \%$ & $-70,0 \%$ & - & $26,9 \%$ & $-9,9 \%$ & $-40,4 \%$ \\
\hline Guatemala & $118,7 \%$ & $-41,7 \%$ & $80,1 \%$ & $19,4 \%$ & $-42,1 \%$ & $-82,6 \%$ & $26,9 \%$ & - & $-76,3 \%$ & $-53,4 \%$ \\
\hline México & $-17,7 \%$ & $-59,5 \%$ & $66,2 \%$ & $18,9 \%$ & $39,7 \%$ & $-43,6 \%$ & $-9,9 \%$ & $-76,3 \%$ & - & $-58,8 \%$ \\
\hline Venezuela & $-7,0 \%$ & $67,9 \%$ & $-13,2 \%$ & $-56,7 \%$ & $14,5 \%$ & $-85,1 \%$ & $-40,4 \%$ & $-53,4 \%$ & $-58,8 \%$ & - \\
\hline
\end{tabular}

Fuente: Elaboración propia.

Nota: valores positivos (negativos) indican que existe un potencial distanciamiento (acercamiento) cultural entre ambos países. 
Tabla 4

Análisis de regresión, EMD distancias culturales actuales

\begin{tabular}{|c|c|c|c|c|c|}
\hline \multirow{2}{*}{ Variable dependiente } & \multicolumn{2}{|l|}{ Dimensión 1} & \multicolumn{2}{|c|}{ Dimensión 2} & \multirow{2}{*}{$\begin{array}{l}\mathrm{R} \\
\text { Cuadrado }\end{array}$} \\
\hline & Coeficiente & Valor-p & Coeficiente & Valor-p & \\
\hline Asertividad &,- 224 &, 043 &, 196 &, 243 &, 523 \\
\hline Colectivismo &,- 095 &, 002 &, 026 &, 477 &, 764 \\
\hline Colectivismo familiar &,- 005 & 922 &,- 136 &, 154 & ,268 \\
\hline Distancia de poder & ,239 &, 000 &,- 297 &, 002 & ,899 \\
\hline $\begin{array}{l}\text { Evitar la } \\
\text { incertidumbre }\end{array}$ &,- 110 &, 063 &,- 162 & 094 &, 553 \\
\hline Igualdad de género &,- 074 & ,232 &, 166 &, 124 &, 405 \\
\hline $\begin{array}{l}\text { Orientación al } \\
\text { desempeño }\end{array}$ &,- 098 &, 101 &,- 232 & 032 & ,603 \\
\hline Orientación al futuro &,- 122 &, 082 &,- 146 & , 195 & ,468 \\
\hline Orientación humana &,- 134 &, 131 &,- 060 &, 664 & 310 \\
\hline
\end{tabular}

Fuente: Elaboración propia. 


\section{Tabla 5}

\section{Análisis de regresión, EMD distancias culturales potenciales}

\begin{tabular}{|c|c|c|c|c|c|}
\hline \multirow{2}{*}{ Variable dependiente } & \multicolumn{2}{|c|}{ Dimensión 1} & \multicolumn{2}{|c|}{ Dimensión 2} & \multirow{2}{*}{$\begin{array}{c}\mathrm{R} \\
\text { Cuadrado }\end{array}$} \\
\hline & Coeficiente & Valor-p & Coeficiente & Valor-p & \\
\hline Asertividad &, 062 &, 479 & 317 &, 013 & ,612 \\
\hline Colectivismo & , 151 & , 012 & ,069 &, 232 &, 639 \\
\hline Colectivismo familiar &, 041 &, 588 &,- 282 &, 013 &, 624 \\
\hline Distancia de poder &,- 187 &, 123 &,- 087 & ,508 & ,328 \\
\hline Evitar la incertidumbre &, 148 & , 040 &,- 112 &, 145 & ,580 \\
\hline Igualdad de género & 061 & 098 &, 136 &, 008 & ,697 \\
\hline $\begin{array}{l}\text { Orientación al } \\
\text { desempeño }\end{array}$ & 160 & ,000 &,- 052 &, 056 & ,915 \\
\hline Orientación al futuro &, 136 &, 012 &,- 102 &, 070 & ,705 \\
\hline Orientación humana & , 179 &, 000 &, 138 &, 000 & 945 \\
\hline
\end{tabular}

Fuente: Elaboración propia. 


\section{Figura 1}

\section{Distancias culturales entre los países}

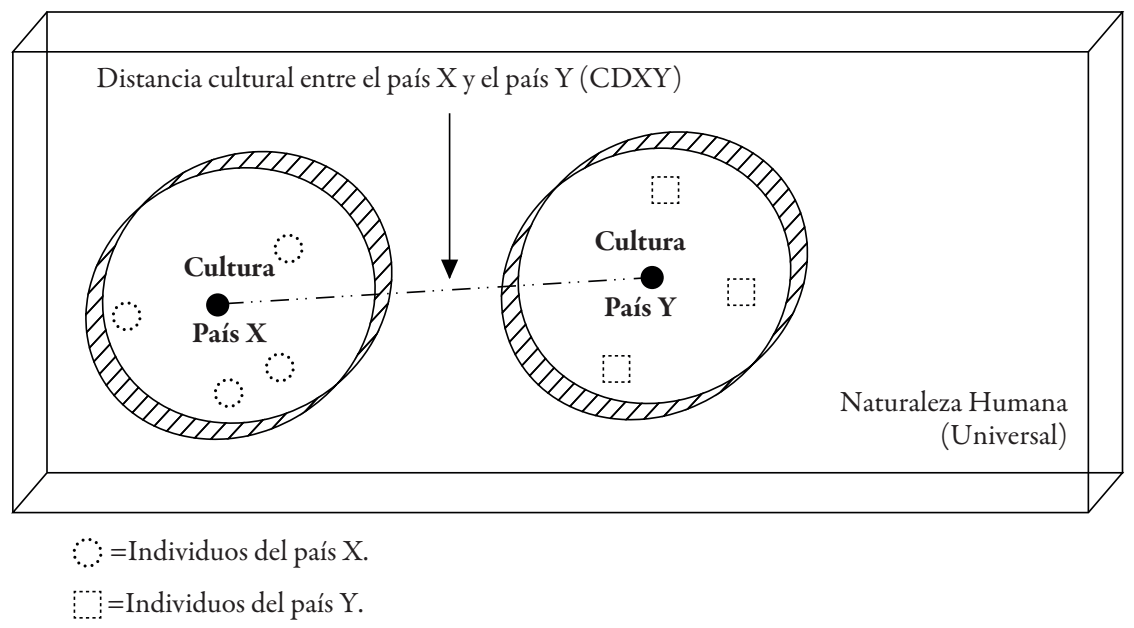

Fuente: Elaboración propia. 
Figura 2

Distancias culturales actuales

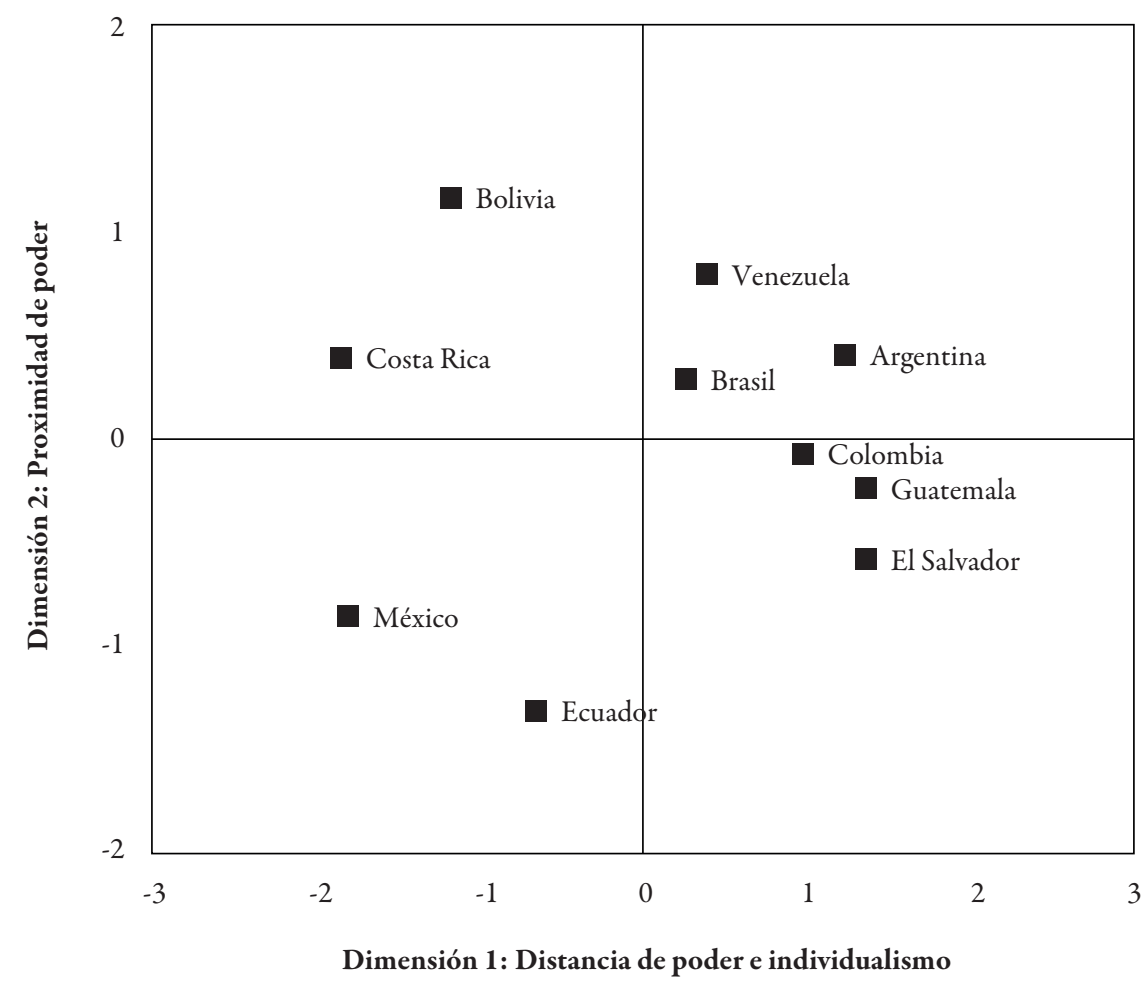

Fuente: Elaboración propia. 
Figura 3

Distancias culturales potenciales

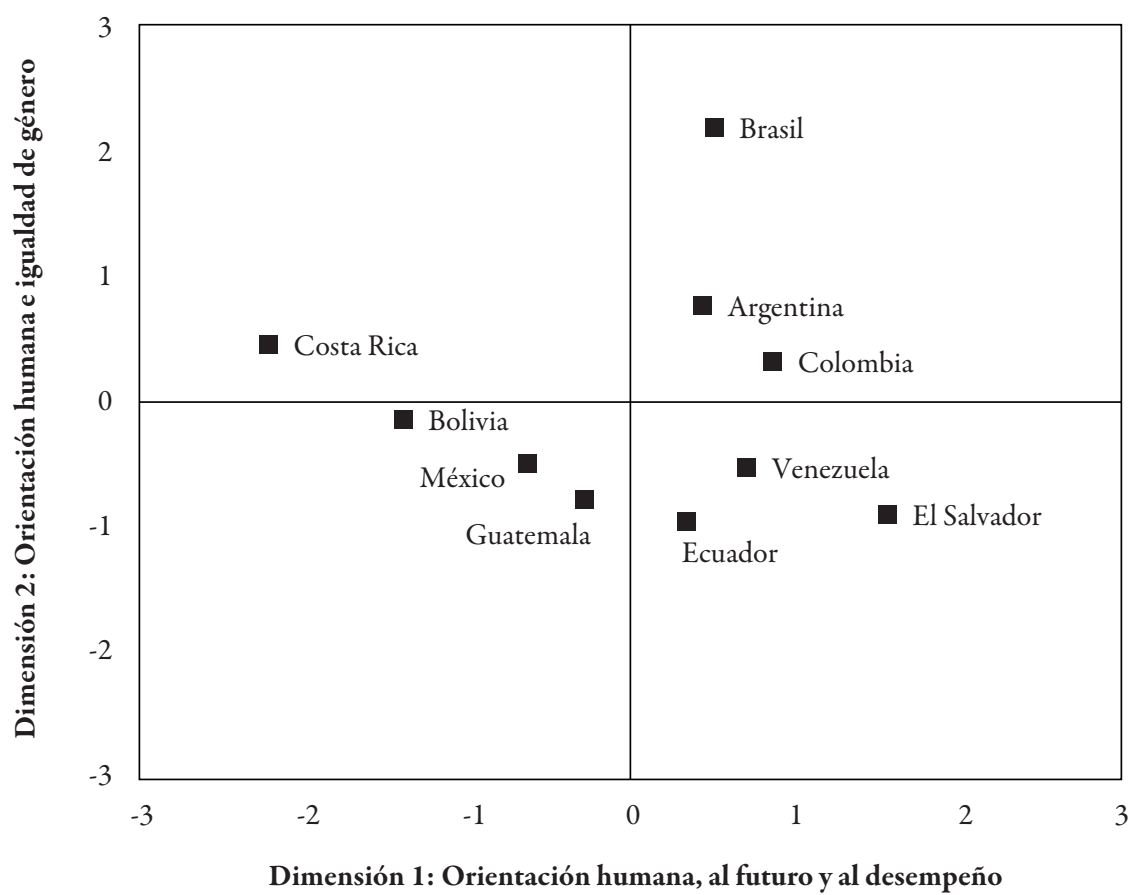

Fuente: Elaboración propia. 


\section{Figura 4}

\section{Distancias culturales actuales y potenciales de México con los 9 países latinoamericanos}

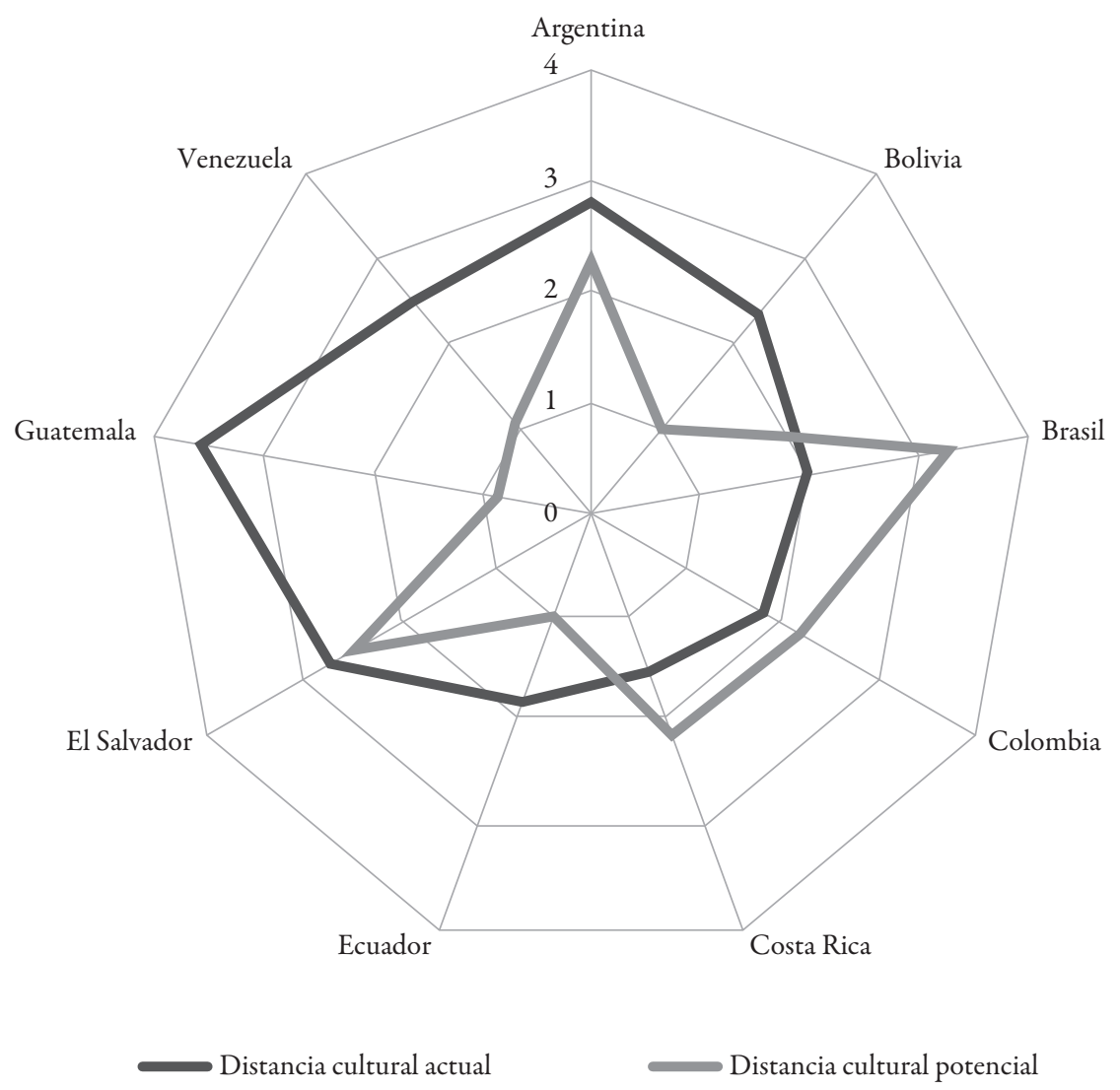

Fuente: Elaboración propia. 


\section{Figura 5}

Distancias culturales actuales y el crecimiento de las exportaciones de México a los 9 países

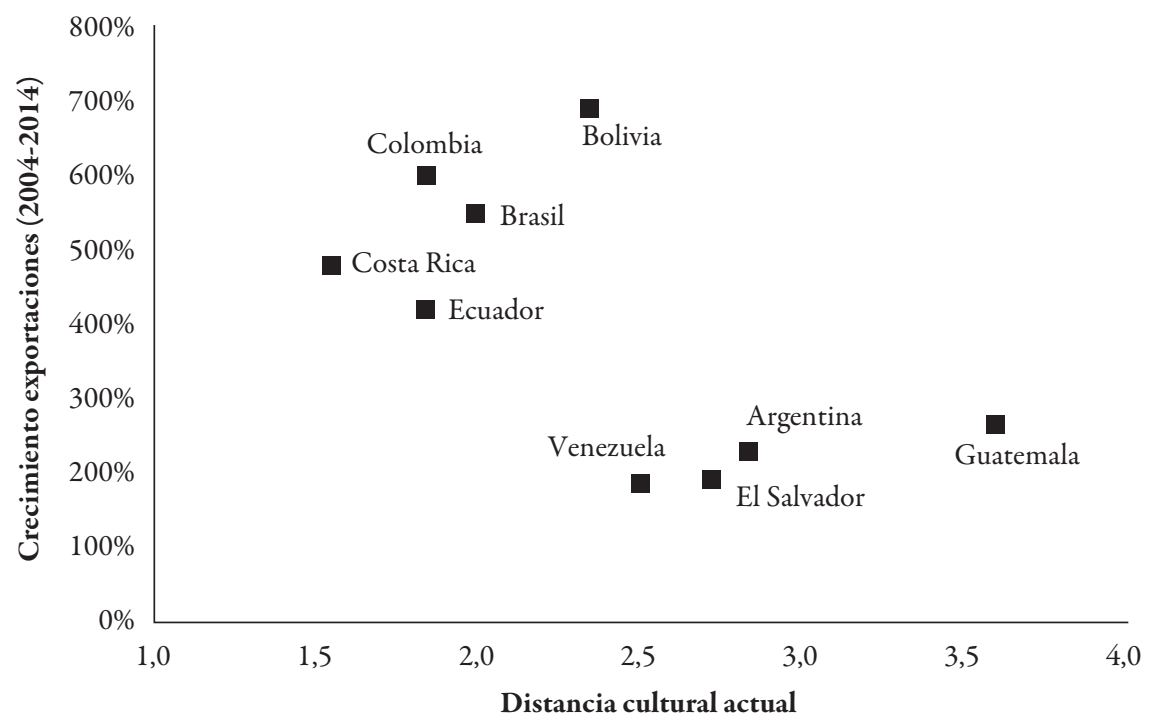

Fuente: Elaboración propia. 


\section{Figura 6}

Distancias culturales actuales y el crecimiento del número de inmigrantes de los 9 países a México

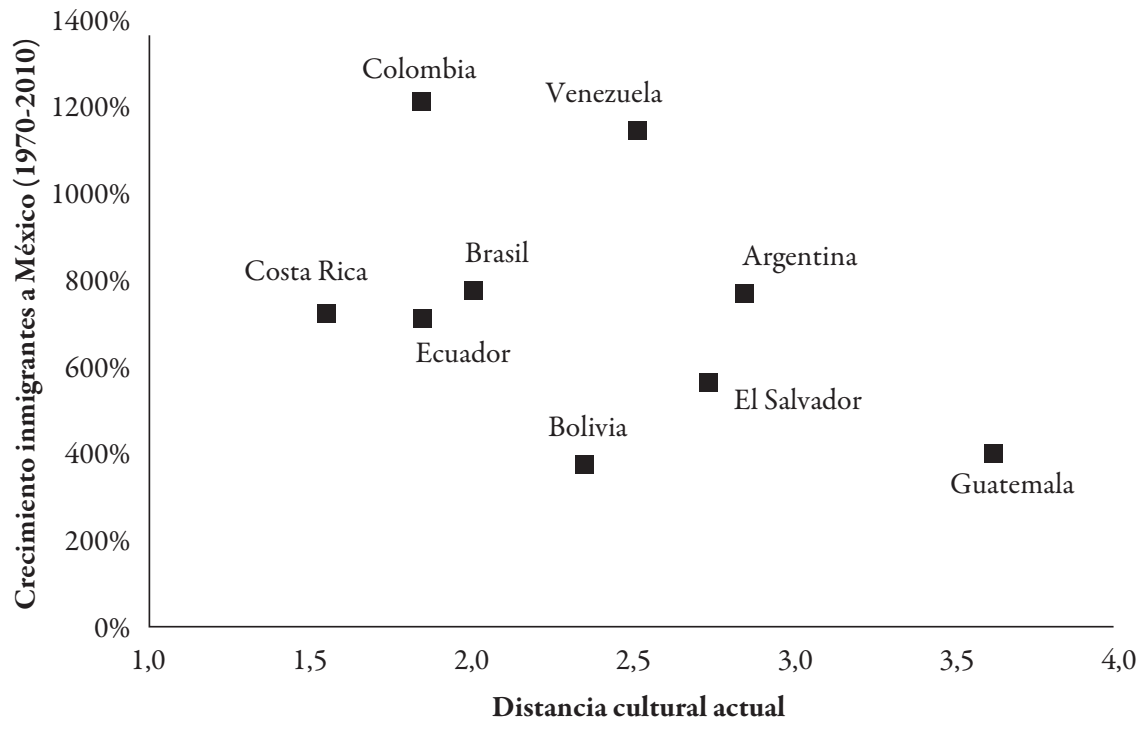

Fuente: Elaboración propia. 


\section{Figura 7}

Distancias culturales potenciales y el número de copublicaciones científicas

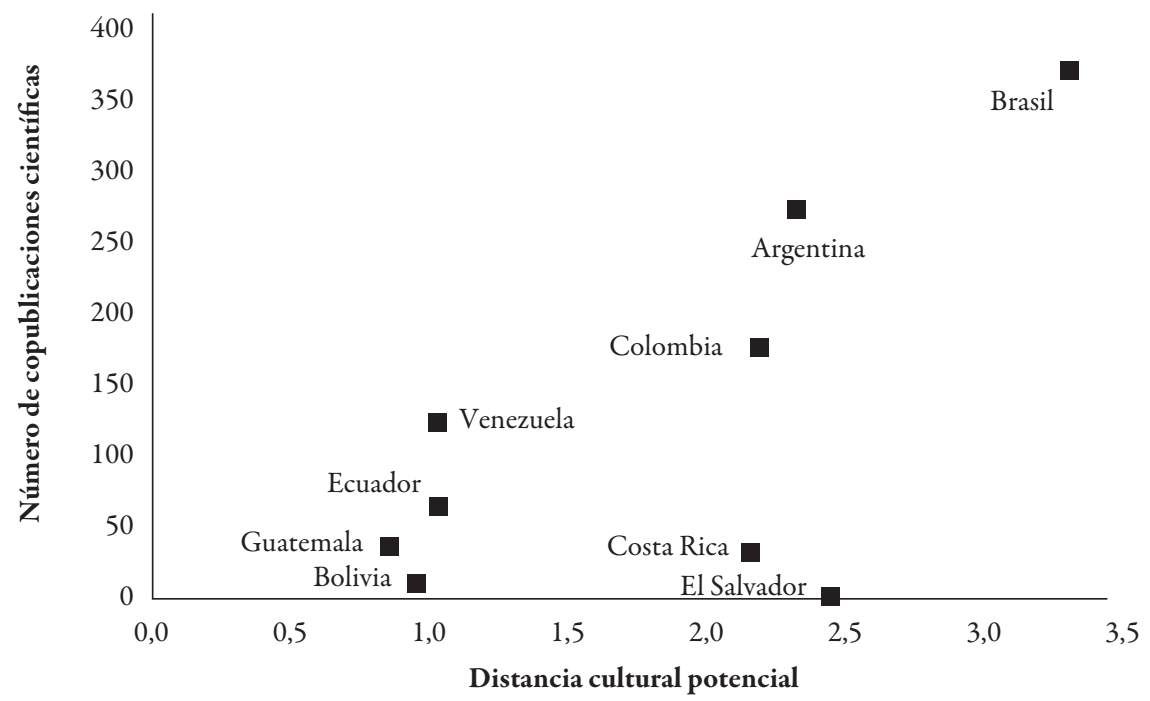

Fuente: Elaboración propia.

Pablo Farías. Doctor en Gestión y Administración de Empresas. Es profesor asistente en la Universidad de Chile, donde imparte los cursos de introducción al marketing, investigación de mercados y métricas de marketing. Líneas de investigación: estrategia de precios, publicidad, marcas, marketing digital, comercio minorista y la toma de decisiones de consumidores y administradores. Publicaciones recientes: Farías, P., "Determinants of the success of global and local brands in Latin America”, en RAE, 55(5) (2015); Farías, P. y R. Rodríguez, "Segment disclosures under IFRS 8's management approach: has segment reporting improved?", en Spanish Journal of Finance and Accounting, $44(2)$ (2015); Valenzuela, L., Torres, E., Hidalgo, P., Farías, P., "Salesperson CLV orientation's effect on performance", en Journal of Business Research, 67(4) (2014).

Recepción: 11 de octubre de 2014.

Aprobación: 5 de septiembre de 2015. 
